\section{Proportional assist: time to re-evaluate a 'new' mode of ventilation for neonates?}

\author{
Andreas Schulze, ${ }^{1}$ Peter G Davis ${ }^{2}$
}

Over the past three decades, a myriad of new ventilatory modalities have been developed for use in neonatal critical care. Most of these modalities are based on a specific physiological rationale. Supportive evidence is sparse and where it exists mostly comes from studies which are shortterm and have a physiological variable as their primary outcome. Randomised controlled trials with clinically important longterm outcomes are rare in this field. In the absence of high quality evidence, the clinical practice of neonatal mechanical respiratory support varies widely between countries, between units and even within units depending on individual preferences.

Bhat et $a l^{1}$ present a cross-over study of Proportional Assist Ventilation (PAV) versus Assist Control Ventilation. Conventional modes of patient-triggered ventilation such as Assist Control typically synchronise a preset ventilator cycle to certain time points of the spontaneous inspiratory effort. For example, the upstroke in ventilator pressure is coupled to the onset of spontaneous inspiration. With all these conventional modes, most clinicians think of the ventilator as a pump. The concept of PAV is fundamentally different; the applied ventilator pressure is continuously coupled to signals derived from the infant's spontaneous breathing. This is achieved by rapid feedback that modulates the ventilator pressure continuously according to the input signals of tidal volume, and/or airflow during spontaneous breathing. The applied ventilator pressure responds to the input signals in a proportionate fashion. Proportionality factors ('gains') are set by the clinician. In this way, the ventilator augments the infant's spontaneous breathing activity while the contour, size and timing remain entirely controlled by the driver, that is, the patient. The ventilator functions like a

${ }^{1}$ Division of Neonatology, Department of Obstetrics and Gynecology, The Children's Hospital, University of Munich, Munich, Germany; ${ }^{2}$ Department of Neonatal Services, The Royal Women's Hospital, University of Melbourne, Parkville, Victoria, Australia

Correspondence to Professor Andreas Schulze, Division of Neonatology, Department of Obstetrics and Gynecology, The Children's Hospital, University of Munich, Marchioninistr.15, Klinikum, Munich D 81377, Germany; andreas.schulze@med.uni-muenchen.de supplemental respiratory muscle under the biological control of breathing feedback-loops.

Servo-controlled systems working on the same principles are ubiquitous in engineering and familiar to users, for example, power-steering in cars and microphoneloudspeaker systems. However, translation of these principles into the clinical practice of respiratory support is made difficult because of the ingrained perception that a ventilator is simply a 'pump'. Consequently, these specific servo-control principles have not become widely used for respiratory support although several investigators who worked independently from one another explored potential clinical applications since almost half a century. Various names were suggested in the literature for the same basic principles: 'Negative ventilator impedance', 'Negative ventilator compliance and resistance', 'Resistive and elastic unloading' and 'Proportional Assist'. ${ }^{2-4}$ Most investigators used pneumotachographic tidal airflow and volume signals of spontaneous breathing as the driving input signals. More recently, a filtered and rectified signal of diaphragmatic electrical activity has been suggested for a proportional amplification technique (neurally adjusted ventilatory assist).

The results of the study by Bhat et al are largely consistent with previous bench studies and short-term clinical investigations of the physiological effects of PAV. It contributes, however, more comprehensive physiological data in a cohort of extremely premature infants with rapid spontaneous respiratory rates and small tidal volumes, that is, technically challenging conditions for a PAV ventilator that must provide a pressure output that adjusts without time lag to the driving signal. It is important to note that while PAV is an effort-augmenting mode, the infants choose small tidal volumes within a range that is currently regarded as protective against volutrauma of the premature lung.

There are some important limitations of this study: The small sample size and short duration of study preclude any assessment of safety of PAV as a long-term mode of respiratory support. Although there is no generally agreed upon gold standard of 'conventional ventilation', the use of pressure limited ventilation as the comparator could be questioned. A recent meta-analysis favoured volume targeting over pressure limited modalities. 5 The early stopping of the trial at 12 of 18 planned patients is problematic. This does not appear to have been a prespecified interim analysis and the decision to stop was taken by the investigators themselves rather than an external committee. The clinical importance of some of the statistically significant but small differences in median airway pressure and peak inflation pressures is doubtful. The use of shouldered, mostly leak-free endotracheal tubes is uncommon among neonatal units and might have implications with respect to generalisability of the results of this study. Large endotracheal tube leaks may impair PAV performance when leak flow causes the ventilator pressure to rise out of proportion to the airflow entering the lung. Ventilator software can be used to distinguish between leak and airflow that truly enters the lung, however, sudden large inspiratory leaks may still cause aberrant ventilator pressure contours touching set upper pressure limits.

In summary, PAV provides a different paradigm for the provision of ventilatory assistance. The useful study by Bhat et al confirms that the technique may change the way we think about and deliver respiratory support to the high-risk population of preterm infants. Over the past decade neonatologists have become more aware of the contribution infants can make to their own ventilation, hence the recent uptake of continuous positive airway pressure as primary therapy even for extremely preterm infants. Given that some infants require more support, the logical next step is to give them more control over the assistance they receive than while on a 'pumping-type' modality. PAV allows them to more finely regulate this assistance and may have the potential to reduce the damage associated with 'conventional' ventilation. Some specific physiological consequences of the technique remain to be explored. The effect of mechanical augmentation of ventilation on control of breathing, apnoea and respiratory muscle dysfunction in infants is of interest. However, the technique needs to be ultimately evaluated in an adequately powered, parallel randomised controlled trial with important clinical outcomes like time on ventilation, time in supplemental oxygen and bronchopulmonary dysplasia.

\section{Competing interests None.}

Provenance and peer review Commissioned; internally peer reviewed. 


\section{Editorial}

To cite Schulze A, Davis PG. Arch Dis Child Fetal Neonatal Ed 2015;100:F4-F5.

Arch Dis Child Fetal Neonatal Ed 2015;100:F4-F5.

Received 11 July 2014

Accepted 8 August 2014

Published Online First 28 August 2014

\section{Linked}

- http://dx.doi.org/10.1136/fetalneonatal-2013305817 doi:10.1136/archdischild-2014-306669

\section{REFERENCES}

1 Bhat P, Patel D-S, Hannam S, et al. Crossover study of proportional assist versus assist control ventilation. Arch Dis Child Fetal Neonatal Ed 2015;100:35-8.

2 Poon CS, Ward SA. A device to provide respiratorymechanical unloading. IEEE Trans Biomed Eng 1986;33:361-5.
3 Schulze A, Schaller P, Gehrhardt B, et al. An infant ventilator technique for resistive unloading during spontaneous breathing. Results in a rabbit model of airway obstruction. Pediatr Res 1990;28:79-82

4 Younes M. Proportional assist ventilation, a new approach to ventilatory support. Theory. Am Rev Respir Dis 1992;145:114-20.

5 Wheeler K, Klingenberg C, McCallion N, et al. Volume-targeted versus pressure-limited ventilation in the neonate. Cochrane Database Syst Rev 2010;(11): CD003666. 